\title{
Clinical implications of STAT6 mRNA and protein knockdown in human colon adenocarcinoma cell line, HT-29
}

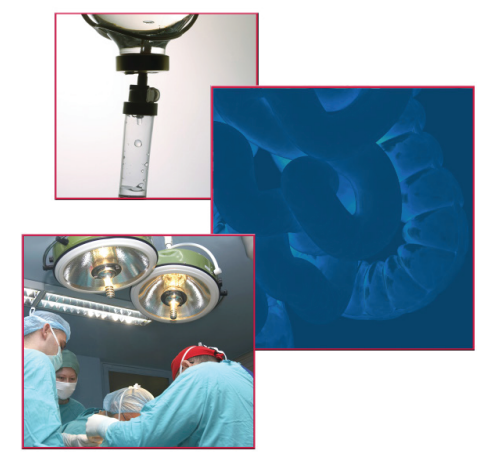

\author{
Carmen Salguero-Aranda ${ }^{\ddagger}, 1$ \& Daniel Sancho-Mensat*,1 (iD) \\ ${ }^{1}$ Celixir plc, Stratford-upon-Avon, UK \\ *Author for correspondence: daniel.sancho@celixir.com \\ $¥$ No longer an employee at Celixir plc. \\ "However, not all that glitters is gold, and siRNAs still need to overcome many challenges before \\ reaching the clinic."
}

First draft submitted: 27 February 2019; Accepted for publication: 7 May 2019; Published online: 13 June 2019

Keywords: cancer $\bullet$ colorectal cancer $\bullet$ gene therapy $\bullet$ molecular oncology $\bullet$ small interfering RNA • STAT6 • targeted drugs

Colorectal cancer (CRC) represents $10.2 \%$ of cancers worldwide, ranking third by incidence in both sexes combined [1]. Approximately 1.8 million new cases were diagnosed worldwide in 2018. It is, overall, the second most common cause of death by cancer globally $(881,000,9.2 \%)$, following lung cancer $(18.4 \%)$, which leads to cancer mortality [1]. The 5-year relative survival rate for patients with stage IIIC and IV colon cancer is approximately 53 and 11\% respectively. Actual CRC treatments involve a multimodal approach based on tumor characteristics and patient-related factors. Conventional treatment remains surgery in combination with a regime of adjuvant chemotherapy. Most patients with CRC metastasis are treated with chemotherapy in combination with targeted drugs (antibodies, small molecule drugs, etc); however, the prognosis in these cases remains poor and the treatment is mainly palliative [2]. Bevacizumab, a humanized monoclonal IgG antibody that targets VEGF-A, is being used in clinical trials. VEGF-A plays a vital role in the regulation of promoting tumor angiogenesis [3]. Moreover, clinical studies have evaluated bevacizumab-based combination therapy in metastatic CRC. A meta-analysis showed that the addition of irinotecan, fluorouracil and leucovorin to bevacizumab better benefits progression-free survival and safety. Addition of leucovorin was associated with better overall response rate and overall survival [4].

Finding new biomarkers and therapeutic targets is vital for the development of personalized and more effective anticancer therapies. The Human Protein Atlas profiles the expression of different proteins in samples of healthy or cancer tissues by means of antibody-based proteomics. High levels of the transcription factor STAT6 have been reported in different types of cancers such as colon, breast, pancreas or lung [5]. In CRC specifically, the aberrant expression of STAT6 has been associated with worse prognosis and malignancy as well as lower survival rates [6]. Other studies have also shown that activation of the IL-13/Il-13Ra1/STAT6/ZEB1 route plays an important role in CRC aggressiveness [7]. Similar observations have also been made in glioblastoma or pancreatic cancer. This makes STAT6 a great candidate for targeted therapies; silencing STAT6 in cases of CRC with high STAT6 expression may have positive effects and help the actual treatments. For all these reasons, an approach for silencing STAT6 expression in STAT6 ${ }^{\text {high }}$ CRCs has the potential to be an effective treatment of those cancers.

\section{Small interfering RNA potential in cancer treatment}

Recent studies have shown that four small interfering RNAs (siRNAs) targeting STAT6 mRNA silenced STAT6 expression, reduced the number of live cells and induced apoptosis in the human cancer cell line, HT-29 [8]. The proliferative capacity of this cell line depends, in part, on signalling via the STAT6 pathway. HT-29 is sensitive to standard treatments oxaliplatin, 5-fluorouracil [9] and bevacizumab [10], and provides a useful model for colorectal cancer therapeutic testing. 
This is not the first time that STAT6 knockdown has been shown to be effective against HT-29 cells. Studies carried out by Zhang et al. showed in 2006 that STAT6-specific short hairpin RNAs (shRNAs) inhibit proliferation and induce apoptosis in HT-29 cells [11]. In this study, HT-29 cell viability was decreased, and the apoptosis analysis indicated that STAT6 shRNAs induced significant early apoptotic events. However, the STAT6 siRNAs used by Salguero-Aranda et al. also have the advantage of inducing late apoptosis [8].

Although both shRNA and siRNA have been proven successful in silencing specific genes, the latter offers several advantages. On the one hand, siRNA sequence can be custom designed or selected from one of the many libraries of predesigned sequences. On the other hand, the simpler structure of siRNAs makes them easier to modify chemically to increase their stability or to add small molecules or fluorochromes. In addition, due to their small size, siRNAs can be delivered directly to the cell without the need for an insertion vector, thus reducing the amount of exogenous DNA introduced into target cells [12]. For these advantages, among others, siRNAs have become one of the most popular tools for genetic therapies.

However, not all that glitters is gold, and siRNAs still need to overcome many challenges before reaching the clinic. Several barriers exist to achieving effective and controlled in vivo delivery which limits siRNA potential. To date, at least 22 RNAi-based drugs have entered clinical trials and intravenous/systemic delivery is the main choice [13]. During its journey to their target tissue, the siRNA complexes have to overcome several physical and chemical barriers, including kidney filtration, phagocytosis, neutralization by serum proteins or degradation by nucleases present in the circulatory system [14]. Therefore, the current delivery methods try to supply the siRNA molecules without affecting their function. These methods are diverse, from lipid- or polymer-based complexes to inorganic nanoparticles to chemically modified naked siRNAs or in conjugation with anticancer drugs [15]. All the above have been tested and achieved effective siRNA delivery into the target cells. Nonetheless, other approaches are possible. Different siRNA formulations have been successfully administered directly into different tissues and organs, avoiding the systemic issues. Compared with systemic injection, local delivery allows lower injection doses and has less dependence on carriers and/or siRNA modifications. Local delivery to the tumor in combination with polymer-based nanoparticles (PEI, chitosan, PEC) has already been proved useful in research, Tang et al. In 2016 successfully administered siRNA through microneedle injection directly in cervical tumor xenografts with effective knockdown of HPV 16 E6 while having minimal adverse effects [16]. We are proposing to use this approach with our STAT6 siRNA sequences to try to ensure efficacy in CRC while avoiding any potential side effects.

\section{STAT6 silencing clinical implications}

It is the author's belief that siRNA therapies will be part of the next generation of anticancer drugs. In this particular case, the potential benefit from an anti-STAT6 siRNA therapy lies in reduction of the tumor size and the reduction of its migration and metastasis capabilities. Although the delivery methods and the pharmacokinetics of the siRNA have not been determined yet, the administration of STAT6 siRNA to CRC patients has the potential to silence and lead to apoptosis of CRC cancer cells and thereby slow down and even halt the tumor growth. This would dramatically increase the quality of life and prognosis of CRC patients.

Early CRC stages (0-I) are often curable with surgery and usually no additional treatment is needed unless the polyp cannot be fully removed. However, the location of the polyps is important, as the pelvis anatomic complexity adds complications to the treatment. For example, rectal tumors are associated with a worse prognosis than colon tumors [17]. In these cases, the potential benefit from anti-STAT6 siRNA administration lies in the possibility of reducing the tumor size, thus facilitating the surgery. In addition, by reducing the tumor metastatic capabilities and triggering cell apoptosis, the chances of recurrence after surgery would likely decrease.

In CRC stages II-III, preventive chemotherapy is often used to reduce tumor size. Although effective, chemotherapy is always associated with many side effects which reduce the quality of life of the patient, and, in some cases, symptoms may persist after the treatment. Moreover, latest studies have suggested that many patients might be overtreated with adjuvant chemotherapy and that current criteria need to be introduced for the selection of patients that would benefit from it [17]. Avoiding the side effects of chemotherapy while maintaining most or all of its benefits would be ideal. Similar to what can be expected for early CRC stages, silencing STAT6 could lead to better prognosis in later stages of CRC as it may reduce the need for chemotherapy, and thus the side effects linked to it, while still reducing tumor size and killing the CRC cells by activating apoptotic events.

It is in the latest stages of CRC (IVa-IVb) where the potential of silencing STAT6 is more uncertain. In these stages, cancer has spread and reached distant organs like the lungs or liver. In these patients, surgical resection, with or without previous downsizing, represents their best chance of survival. However, this is only applicable to a limited 
number of patients. When patients present unresectable metastases, systemic therapy is needed, combining two or more chemotherapies and/or targeted drugs, such as bevacizumab. Systemic treatment significantly prolongs overall survival and might allow for resection [17]. Here, STAT6 silencing could be introduced in combination with the mentioned therapies, aimed at downsizing the tumors and favoring resection. As discussed before, STAT6 expression in CRC is correlated with poorer prognosis and higher aggressiveness of the cancer. Thus, STAT6 screening should be considered before deciding the line of treatment in CRC patients. For STAT6 ${ }^{\text {high }}$ CRC patients, our new STAT6 siRNA treatment could mean the difference between life or death.

\section{Conclusion}

While it is true that anti-cancer therapies have improved over the past few years, there is still a long way to go. siRNA-based therapies represent a powerful tool in the fight against cancer. Oncogenes, mutated tumor suppressor genes or any other gene related to cancer are great candidates for this type of therapy. The STAT6 transcription factor is a potential target, as its silencing has been proven to halt cancer progression both in vitro and in vivo. Being related with poor prognosis and survival in CRC, the silencing of STAT6 could result in increased resectability and, thus fewer doses of chemotherapy. Nonetheless, progress still needs to be made and more robust data of its efficacy in clinical trials is needed.

\section{Financial \& competing interests disclosure}

The authors have received financial support from their employer, Celixir plc, in form of salaries during the creation of this manuscript. The commercial affiliation of the authors with Celixir plc does not alter his adherence to Future Medicines policies on sharing materials and data. The authors have no other relevant affiliations or financial involvement with any organization or entity with a financial interest in or financial conflict with the subject matter or materials discussed in the manuscript apart from those disclosed.

No writing assistance was utilized in the production of this manuscript.

\section{Open access}

This work is licensed under the Attribution-NonCommercial-NoDerivatives 4.0 Unported License. To view a copy of this license, visit http://creativecommons.org/licenses/by-nc-nd/4.0/

\section{References}

Papers of special note have been highlighted as: $\bullet$ of interest; $\bullet \bullet$ of considerable interest

1. Bray F, Ferlay J, Soerjomataram I, Siegel RL, Torre LA, Jemal A. Global cancer statistics 2018: GLOBOCAN estimates of incidence and mortality worldwide for 36 cancers in 185 countries. CA. Cancer J. Clin. 68(6), 394-424 (2018).

2. Mármol I, Sánchez-de-Diego C, Pradilla Dieste A, Cerrada E, Rodriguez Yoldi M. Colorectal carcinoma: a general overview and future perspectives in colorectal cancer. Int. J. Mol. Sci. 18(1), 197 (2017).

- Review of the landscape in colorectal cancer (CRC) diagnosis, treatment and future perspectives.

3. Ferrara N. Vascular endothelial growth factor: basic science and clinical progress. Endocr. Rev. 25(4), 581-611 (2004).

4. Xu R, Xu C, Liu C, Cui C, Zhu J. Efficacy and safety of bevacizumab-based combination therapy for treatment of patients with metastatic colorectal cancer. OncoTargets Ther. 11, 8605-8621 (2018).

5. Uhlén M, Björling E, Agaton C et al. A human protein atlas for normal and cancer tissues based on antibody proteomics. Mol. Cell. Proteomics 4(12), 1920-1932 (2005).

6. Wang C-G. EZH2 and STAT6 expression profiles are correlated with colorectal cancer stage and prognosis. World J. Gastroenterol. 16(19), 2421 (2010).

-. These two papers highlight the role that the STAT6 pathway plays in cancer development.

7. Cao H, Zhang J, Liu H et al. IL-13/STAT6 signaling plays a critical role in the epithelial-mesenchymal transition of colorectal cancer cells. Oncotarget 7(38), 61183-61198 (2016).

-. These two papers highlight the role that the STAT6 pathway plays in cancer development.

8. Salguero Aranda C, Sancho Mensat D, Sultan S, Reginald A, Chapman L. STAT6 knockdown using multiple siRNA sequences inhibits proliferation and induces apoptosis of human colorectal and breast cancer cell lines. PLoS ONE 14(5), 0207558 (2019).

9. Flis $S$, Spławiński J. Inhibitory effects of 5 -fluorouracil and oxaliplatin on human colorectal cancer cell survival are synergistically enhanced by sulindac sulfide. Anticancer Res.29(1), 435-441 (2009).

10. Hein M, Graver S. Tumor cell response to bevacizumab single agent therapy in vitro. Cancer Cell Int. 13(1), 94 (2013).

- Example of the use of RNA interference technology for STAT6 silencing in CRC. 
11. Zhang M, Zhou Y, Zhang W et al. Apoptosis induced by short hairpin RNA-mediated STAT6 gene silencing in human colon cancer cells. Chin. Med. J. 119(10), 801-808 (2006).

- $\quad$ RNA interference technologies overview.

12. Rao DD, Vorhies JS, Senzer N, Nemunaitis J. siRNA vs. shRNA: similarities and differences. Adv. Drug Deliv. Rev. 61(9), 746-759 (2009).

13. Xu C, Wang J. Delivery systems for siRNA drug development in cancer therapy. Asian J. Pharm. Sci. 10(1), 1-12 (2015).

14. Whitehead KA, Langer R, Anderson DG. Knocking down barriers: advances in siRNA delivery. Nat. Rev. Drug Discov. 8, 129 (2009).

-• Great review that explains the actual landscape in small interfering RNA-based treatments in cancer therapy.

15. Singh A, Trivedi P, Jain NK. Advances in siRNA delivery in cancer therapy. Artif. Cells Nanomed. Biotechnol. 46(2), 274-283 (2018).

16. Tang T, Deng Y, Chen J et al. Local administration of siRNA through microneedle: optimization, bio-distribution, tumor suppression and toxicity. Sci. Rep. 6(1), 30430 (2016).

- This review brings together the latest advances in CRC treatments, focusing in personalized and precision therapies and their advantages/disadvantages compared to actual treatments.

17. Punt CJA, Koopman M, Vermeulen L. From tumour heterogeneity to advances in precision treatment of colorectal cancer. Nat. Rev. Clin. Oncol. 14(4), 235-246 (2017). 